\title{
Pequeñas cosas, grandes problemas. Análisis de hallazgos de dimensiones reducidas en el Mediterráneo oriental antiguo
}

\author{
Small things, big problems. Analysis of small objects in the ancient Eastern \\ Mediterranean
}

\author{
Liliana M. Manzi * \\ Rodrigo Cabrera** \\ Eva Amanda Calomino ${ }^{* * *}$ \\ María Laura lamarino***
}

\section{RESUMEN}

Este trabajo se focaliza en el estudio de pequeños objetos hallados en sitios del Mediterráneo oriental antiguo, con el fin de reevaluar las prácticas sociales y contextos de depositación en los que estuvieron insertos. Debido a sus reducidas dimensiones, los pequeños objetos conforman una clase de artefactos con alto potencial para el estudio de los ámbitos de uso -domésticos y públicos- en los que participaron. Las muestras analizadas en cada uno de los cuatro casos aquí presentados proceden de colecciones con diversos orígenes. En relación a ellos, se cuenta con un historial de expolios y excavaciones no sistemáticas realizadas principalmente entre mediados del siglo XIX y comienzos del XX, las cuales llevaron a que ciertos registros se presentaran en buena medida alterados.

\footnotetext{
* Doctora en Arqueología por la Universidad de Buenos Aires. Investigadora Independiente del Consejo Nacional de Investigaciones Científicas y Técnicas con lugar de trabajo en el Instituto Multidisciplinario de Historia y Ciencias Humanas. Profesora Adjunta de la cátedra "Modelos y Métodos en Economía Prehistórica" en el Departamento de Ciencias Antropológicas de la Universidad de Buenos Aires, Argentina. ORCID: https://orcid.org/0000-0003-0227776X. Correo electrónico: Im_manzi@yahoo.com.ar

** Doctorando en Historia por la Universidad de Buenos Aires. Licenciado en Historia por la misma casa de estudios, donde se desempeña como docente en la cátedra de "Historia Antigua I (Oriente)". Ha sido becario doctoral por el Consejo Nacional de Investigaciones Científicas y Técnicas con lugar de trabajo en el Instituto Multidisciplinario de Historia y Ciencias Humanas, Argentina. ORCID: https://orcid.org/0000-0002-7299-6068. Correo electrónico: cabrera.pertusatti@gmail.com

*** Doctora en Arqueología por la Universidad de Buenos Aires. Becaria posdoctoral por el Consejo Nacional de Investigaciones Científicas y Técnicas con lugar de trabajo en el Instituto Multidisciplinario de Historia y Ciencias Humanas, Argentina. ORCID: https://orcid.org/0000-0001-5013-4996. Correo electrónico: calomino.eva@gmail.com **** Doctoranda en Arqueología por la Universidad de Buenos Aires. Licenciada en Historia por la misma casa de estudios. Becaria doctoral por el Consejo Nacional de Investigaciones Científicas y Técnicas con lugar de trabajo en el Instituto Multidisciplinario de Historia y Ciencias Humanas, Argentina. ORCID: https://orcid.org/0000-0001-69979817. Correo electrónico: mlauiamarino@gmail.com
} 
Palabras clave: pequeños objetos, Mediterráneo oriental, Mesopotamia, Egipto, ámbito doméstico, ámbito público

\section{ABSTRACT}

This article focuses on the study of small objects found in ancient Eastern Mediterranean sites, to reevaluate the social practices and deposition contexts in which they were inserted. Due to their reduced dimensions, the small objects make up a class of artifacts with high potential for studying the areas of use -domestic and public- in which they participated. The samples analyzed in each of the four cases presented here come from collections with different origins. In relation to them, there is a history of looting and non-systematic excavations carried out mainly between the middle of the $19^{\text {th }}$ century and the beginning of the $20^{\text {th }}$, which led to certain records being presented to a large extent altered.

Keywords: small objects, Eastern Mediterranean, Mesopotamia, Egypt, domestic context, public domain Recibido: septiembre 2020

Aceptado: marzo 2021

\section{Introducción}

El estudio de pequeños objetos que proponemos realizar comprende una línea de trabajo que alcanzó su mayor relevancia en la década de los setenta, al demostrar su aplicabilidad en el análisis de materiales considerados perdidos u olvidados y contribuir en el reconocimiento y la discusión de sus contextos de hallazgo. Esta situación, en particular, expuso su alto potencial para analizar registros que se encuentran en buena medida perturbados, abriendo la posibilidad de reconocer y discutir aspectos vinculados con la praxis social en la que estuvieron inmersos.

Desde una perspectiva depositacional y postdepositacional, las dimensiones reducidas de esta clase de evidencia, que le otorgan una alta capacidad de ocultamiento, resultan de sumo interés, puesto que facilitaron su persistencia en los lugares de depositación. Asimismo, al conformar una unidad discreta y compacta, a pesar de perder algunos de sus apéndices o salientes, se mantienen morfológicamente reconocibles. En algunos casos, por encontrarse en sus posiciones originales de uso, almacenamiento o descarte, permiten reconocer los procesos que terciaron para que eso ocurriera, tales como posibles pérdidas, abandonos, equipamientos de lugares y/o residuos de facto. En tanto, los episodios de redepositación dan cuenta de su remoción a partir de contextos primarios, resultando desplazados para su disposición final ${ }^{1}$.

\footnotetext{
${ }^{1}$ Allison, Penelope. 1999. The Archaeology of Household Activities, London, Routledge; Schiffer, Michael. 1987. Formation Processes of the Archaeological Record, Albuquerque, The University of New Mexico Press; Sharer, Robert \& Wendy Ashmore. 2002. Archaeology: Discovering Our Past, Boston, Mc Graw-Hill.
} 
Nuestra propuesta apunta a la comprensión de las prácticas sociales en las que los pequeños objetos estuvieron involucrados, dado que, en función de sus tamaños, alcanzaron cierta estabilidad depositacional, resistiendo a diversas perturbaciones ${ }^{2}$. De este modo, una relectura de los procesos de corta, mediana y larga duración, en los que se desenvolvieron las biografías artefactuales aquí consideradas, posibilita un reconocimiento de las circunstancias de producción, circulación, almacenamiento y/o descarte de los objetos.

La práctica social no comprende exclusivamente entidades binómicas y, más allá del lugar de depositación de los pequeños hallazgos, es posible que muchos de ellos interactuaran de múltiples formas y participaran tanto de los ámbitos públicos como privados. Los primeros aluden a órbitas oficiales, sean de corte secular o religioso, con incidencia colectiva. Los segundos, generadas de forma privada o personal, son representativos de decisiones individuales, o bien de las unidades domésticas y/o del linaje ${ }^{3}$.

Las muestras presentadas provienen de contextos arqueológicos del Mediterráneo oriental: a) Baja Mesopotamia, actual Irak (2110-2003 a.C.); b) Tell el-Ghaba en el Norte de Sinaí (mediados del siglo $\mathrm{X}$ a fines del siglo VII a.C.); c) Tell el-Amarna (1350-1335 a.C.) y $d$ ) Tebas Occidental (1550-1307 a.C.) en Egipto. El área, desde una perspectiva geográfica, integra el extremo oeste de Europa, norte de África y este de Asia como unidad relevante para el estudio de las sociedades antiguas. Por medio de este constructo, adherimos a aquellas perspectivas históricas y culturales que plantean una superación de las divisiones preexistentes entre Occidente y Oriente, o entre un mundo europeo y otro propiamente asiáticoafricano.

En una propuesta inicial, Braudel realizó un abordaje particular para el estudio de la región mediterránea con la meta de integrar procesos socioculturales de "larga duración"4. Posteriormente, Said se refirió a las sociedades del Mediterráneo oriental a partir del criterio de dominación que algunas potencias europeas -en principio, Inglaterra y Francia- ejercieron en el área desde el siglo XVII y que se corrobora a partir de los escritos de algunos intelectuales

\footnotetext{
2 Nielsen, Axel. 1987. "Trampling the Archaeological Record: an Experimental Study", en American Antiquity, vol. 53, no 3, Washington, pp. 483-503; Nielsen, Axel. 1991. "Se hace camino al andar. Estudio experimental sobre patrones de fractura en material cerámico sometido a pisoteo", en Shincal, Vol. 3, № 2, San Fernando del Valle de Catamarca, pp. 116-139; Politis, Gustavo. 2000. "La formación de sitios de sitios de cazadores-recolectores en las Tierras Bajas Sudamericanas: un caso de estudio etnoarqueológico", en Alicia Durán Coirolo \& Roberto Bracco Boksa (Eds.), Arqueología de las Tierras Bajas, Montevideo, Ministerio de Educación y Cultura, Comisión Nacional de Arqueología, pp. 250-266.

${ }^{3}$ Banton, Michael. 1965. Roles: An Introduction to the Study of Social Relations, London, Tavistock; Sahlins, Marshall. 1972. Stone Age Economics, New York, de Gruyter; Gillespie, Susan D. 2007. “When is a House?”, en Robin Beck (Ed.), The Durable House: House Society Models in Archaeology (Occasional Paper 35), Carbondale, Center for Archaeological Investigations, pp. 25-50.

${ }^{4}$ Braudel, Fernand. 1987 (1949). El Mediterráneo y el mundo mediterráneo en la época de Felipe II, México, Fondo de Cultura Económica, p. 27; Braudel Fernand. 1958. "Histoire et Sciences sociales: La longue durée", en Annales.

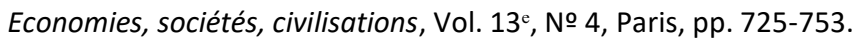


que podemos denominar como "orientalistas" 5 . Nos referiremos aquí, en particular, a dos formaciones estatales: Mesopotamia -con base territorial en los actuales Siria e Irak- y Egipto -en el espacio que ocupa la nación homónima, Sudán y parte del corredor sirio-palestinense.

Desde inicios del siglo XIX hasta el presente, en los territorios mencionados, tuvieron lugar innumerables saqueos, producto de potencias extranjeras principalmente que los despojaron de sus patrimonios histórico-culturales y afectaron su preservación ${ }^{6}$. De forma paralela, diversos procesos ocupacionales locales profundizaron la pérdida de los contextos de hallazgo y promovieron la dispersión de piezas arqueológicas al estar dirigidos, entre otras posibilidades, a la obtención y reutilización de materiales para la construcción y el empleo de algunas estructuras como residencias ${ }^{7}$. Finalmente, durante el último medio siglo, la inestabilidad política y los escenarios de conflicto bélico agravaron esta tendencia ${ }^{8}$.

En este marco de múltiples y profundas perturbaciones, enfatizamos el hallazgo de pequeños objetos, los cuales fueron también foco de interés dada su transportabilidad y facultad de convertirse en piezas coleccionables. Por lo expuesto, y porque responden a una multiplicidad de prácticas, consideramos que su análisis permite reconstruir procesos de depositación, contextos de hallazgo y ámbitos de los que fueron parte.

\section{El tratamiento de los pequeños objetos en el Mediterráneo oriental antiguo}

En In Small Things Forgotten, Deetz ${ }^{9}$ refiere por primera vez a la idea de que los pequeños objetos pudieron tener un rol importante en la vida de las personas y destaca su relevancia en las investigaciones arqueológicas. De acuerdo con el autor, ciertas propuestas clasificatorias fueron construidas a partir de evidencia arqueológica con prescindencia de registros documentales -a pesar de estar disponibles- $y$, al cotejarlas, son notables las diferencias que guardan con las expectativas y los fines que adoptaron para quienes manufacturaron y usaron esos objetos.

\footnotetext{
${ }^{5}$ Said, Edward. 2002 (1978). Orientalismo, Barcelona, Debolsillo, pp. 98-99.

${ }^{6}$ Colla, Elliott. 2007. Conflicted Antiquities. Egyptology, Egyptomania, Egyptian Modernity, Durham/London, Duke University Press; Mitchell, Timothy. 1988. Colonising Egypt, Berkerley/Los Angeles/London, University of California Press.

7 Vivant Denon, Dominique. 2005 (1802). Sin Mañana. Viaje al Bajo y Alto Egipto durante las campañas del general Bonaparte, Madrid, Atalanta; Davies, Norman de Garis. 1933. The Tomb of Neferhotep at Thebes, New York, Metropolitan Museum of Art; Trigger, Bruce. 1992 (1989). Historia del pensamiento arqueológico.

8 Simpson, Caroline. 2003. "Modern Qurna-Pieces of an Historical Jigsaw", en Nigel Strudwick \& John Taylor (Eds.), Present and Past in the Theban Necropolis, London, The British Museum Press, pp. 244-249; Spence, Kate. 2015. "Ancient Egyptian Houses and Households: Architecture, Artefacts, Conceptualization and Interpretation", en Miriam Müller (Ed.), Household Studies in Complex Societies. (Micro)Archaeological and Textual Approaches, Chicago, University of Chicago Press, pp. 83-99; van der Spek, Kees. 2011. The Modern Neighbors of Tutankhamun: History, Life, and Work in the Villages of the Theban West Bank, Cairo/New York, The American University in Cairo Press; Molina, Manuel. 2020. "The Looting of Ur III Tablets after the Gulf Wars", pp. 323-352.

9 Deetz, James. 1977. In Small Things Forgotten: The Archaeology of Early American Life, New York, Anchor Press.
} 
Asimismo, se mencionan otros aspectos que con posteridad se convirtieron en temas de estudios específicos. Se hizo foco en las formas de distribución de los artefactos (si se presentan concentrados, dispersos o aislados) o si se observan sobre las superficies del terreno o cubiertos por sedimentos ${ }^{10}$. El carácter acresional ${ }^{11}$ de los depósitos arqueológicos ${ }^{12}$ conlleva a que los materiales representados puedan responder a distintas cronologías y manifestarse bajo diferentes formas de "palimpsestos"13, algunos de los cuales no solamente refieren a la mezcla de materiales sino a cuestiones interpretativas.

En concordancia con los procesos que atraviesan los materiales, desde su manufactura hasta su disposición final ${ }^{14}$, pueden trazarse las "biografías" o "historias de vida" de los objetos $^{15}$. En función de los diferentes momentos de uso y descarte que representan un "tiempo transgresivo"16, en el sentido de las perduraciones que se observan y que pueden remitir a la noción de "palimpsesto" ${ }^{17}$. Sobre este particular, la disponibilidad de registros documentales redunda en cronologías más precisas ${ }^{18}$ que las que operan directamente sobre la evidencia material. Esta situación se constata, en especial, en aquellos contextos donde la resolución cronológica tiene una corta resolución temporal, comprendiendo unos años o incluso algunas décadas. Estos rangos temporales se corresponden con tiempos históricos y/o etnográficos, donde las fechas obtenidas a partir de otros métodos de datación presentan un

\footnotetext{
${ }^{10}$ Ebert, James. 1992. Distributional Archaeology, Albuquerque, University of New Mexico Press.

${ }^{11}$ Stern, Nicola. 1994. "The Implications of Time-Averaging for Reconstructing the Land-Use Patterns of Early ToolUsing Hominids", en Journal of Human Evolution, vol. 27, № 1-3, Amsterdam, pp. 89-105.

12 Stein, Julie. 1987. "Deposits for Archaeologists", en Michael Schiffer (Ed.), Advances in Method and Theory, vol. 11, New York, Academic Press, pp. 337-395.

${ }^{13}$ Bailey, Geoff. 2007. "Time Perspectives, Palimpsests and the Archaeology of Time", en Journal of Anthropological Archaeology, vol. 26, № 2, Amsterdam, pp. 198-223.

${ }^{14}$ Schiffer, Michael. 1987. Formation Processes of the Archaeological Record.

${ }^{15}$ Appadurai, Arjun. 1986. "Introduction: Commodities and the Politics of Value", en Arjun Appadurai (Ed.), The Social Life of Things: Commodities in Cultural Perspective, New York, Cambridge University Press, p. 36.

${ }^{16}$ Stern, Nicola. 1994. "The Implications of Time-Averaging for Reconstructing the Land-Use Patterns of Early ToolUsing Hominids", pp. 89-105.

${ }^{17}$ Randall, Henry. 1934. "History in the Open Air", en Antiquity, Vol. 8, № 29, Durham, pp. 5-23; Crawford, Osbert. 1953. Archaeology and the Field, London, Phoenix House Ltd; Binford, Lewis. 1981. "Behavioral Archaeology and the 'Pompeii Premise'", en Journal of Anthropological Research, vol. 37, Albuquerque, NM, pp. 195-208; Bailey, Geoff. 2005. "Concepts of Time", en Colin Renfrew \& Paul Bahn (Eds.), Archaeology: The Key Concepts, London/New York, NY, Routledge, pp. 200-203; Bailey, Geoff. 2007. "Time Perspectives, Palimpsests and the Archaeology of Time", en Journal of Anthropological Archaeology, Vol. 26, № 2, Amsterdam, pp. 198-223; Sullivan, Alan. 2008. "Time Perspectivism and the Interpretive Potential of Palimpsests: Theoretical and Methodological Considerations of Assemblage Formation History and Contemporaneity", en Simon Holdaway \& LuAnn Wandsnider (Eds.), Time in Archaeology: Time Perspectivism Revisited, Salt Lake City, UT, University of Utah Press, pp. 31-45; Lucas, Gavin. 2010. "Time and the Archaeological Archive", en Rethinking History. The Journal of Theory and Practice, vol. 14, no 3, London, pp. 343-359; Cabrera, Rodrigo y Salem, Leila. 2020. "Archivos, palimpsestos y materialidad funeraria. Una discusión en Asiriología y Egiptología a partir de los Estudios de la Cultura Material", Cuadernos de la Facultad de Humanidades y Ciencias Sociales. Universidad Nacional de Jujuy, no 57, San Salvador de Jujuy, pp. 147-175.
}

18 Deetz, James. 1977. In Small Things Forgotten. 
error mayor al que puede contener, por ejemplo, la conversión entre listas dinásticas y sistemas calendáricos.

En el estudio de poblaciones estatales, como las del Mediterráneo oriental antiguo, las investigaciones hicieron foco en la reconstrucción del patrón de asentamiento, reconocer la diversidad de entierros y describir un conjunto de recintos diversos y estructuras edilicias particulares ${ }^{19}$. En la mayor parte de esas publicaciones, prima el carácter monumentalista de sus enfoques y la alta estima por la búsqueda y la recuperación de aquellas piezas mejor conservadas, raras o valiosas, para la provisión de museos y colecciones privadas ${ }^{20}$. Un trato semejante fue dado a los objetos que eran portadores de inscripciones y/o iconografía. En cambio, la evidencia fragmentada o que no resultaba atractiva para su exposición o para ser conservada era infravalorada o rechazada.

Al mismo tiempo, se observan distintas clases de tratamiento con respecto a los materiales. A propósito, en el caso de los procedentes de ámbitos domésticos, resultaron subvalorados, independientemente de que fuesen representativos de la no-élite o de la élite. En términos comparativos, también contaron con una menor valoración que los recuperados en tumbas de la élite, a pesar de que forman parte de las esferas privadas en relación con las denominadas "grandes organizaciones" como los templos y los palacios ${ }^{21}$.

\section{Materiales y contextos}

En este trabajo, nuestra atención se orienta a una clase de evidencia que se destaca por sus dimensiones pequeñas, entre los que se incluyen piezas fragmentadas. Reconocemos las diferencias existentes entre elementos que tienen entidad en sí mismos, tales como adornos, amuletos, objetos identitarios y suntuarios, y otros artefactos que presentan dimensiones pequeñas en el momento de ser descartados o resultar depositados. Cabe indicar que los contextos de depositación de donde provienen los pequeños hallazgos corresponden a estructuras que desempeñaron distintas funciones, como templos, palacios, tumbas y recintos habitacionales.

\footnotetext{
${ }^{19}$ Bard, Kathryn. 1997. "Urbanism and the Rise Of Complex Society And The Early State In Egypt”, en Linda Manzanilla (Ed.), Emergence and Change in Early Urban Societies, New York, Plenum, pp. 59-86; Bard, Kathryn. 2017. "Political Economies of Predynastic Egypt and the Formation of the Early State", en Journal of Archaeological Research, Vol. 25, № 1, New York, pp. 1-36; Wengrow, David. 2006. The Archaeology of Early Egypt. Social Transformations in North-East Africa, 10,000-2650 BC, Cambridge, Cambridge University Press.

20 Moreno, Juan. 2014. "The Cursed Discipline? The Peculiarities of Egyptology at the Turn of the Twenty-First century", en William Carruthers (Ed.), Histories of Egyptology: Interdisciplinary Measures, London/New York, Routledge, p. 58.

${ }^{21}$ Giddy, Lisa. 1999. The Survey of Memphis III. Kom Rabi'a: the New Kingdom and Post-New Kingdom Objects, London, The Egypt Exploration Society/Cromwell Press; Kemp, Barry, Anna Stevens \& Paul Buckland. 2010. Busy Lives at Amarna: Excavations in the Main City (Grid 12 and the House of Ranefer, N49. 18), London, Egypt Exploration Society.
} 
Cada una de las muestras analizadas fueron recuperadas mediante metodologías disímiles, muchas de las cuales se encuentran fuera de nuestra órbita de control (Mesopotamia y Amarna), o bien, proceden de investigaciones previas (con bases de datos preexistentes) que han sido retomadas por nuestras investigaciones en curso. En este sentido, hemos tratado de analizar la evidencia privilegiando las variables nominales -clases de materiales- y ordinales. De acuerdo a una discriminación por tamaños, altamente sustentada sobre bases intuitivas, en la bibliografía se constata de manera implícita una valoración dicotómica entre las grandes estructuras y la evidencia material de menor tamaño, no solo con respecto a los pequeños objetos sino también a la arquitectura ${ }^{22}$.

En este trabajo, agrupamos como pequeños objetos a aquellos hallazgos que tienen $c .4 \mathrm{x}$ $4 \mathrm{~cm}$, en piezas más o menos cuadrangulares, y c. $5 \times 2,5 \mathrm{~cm}$ en ítems de forma rectangular o cilíndrica. Consideramos que, por sus dimensiones menores, cuando forman parte del arreglo personal, refieren a adornos; cuando son objeto de devoción, califican como amuletos; al dar cuenta de identidades o pertenencias, se consideran insignias; si están vinculados a un objeto de mayores dimensiones y refieren a su contenido, pueden ser denominados etiquetas.

Consideramos que, si estos artefactos se presentan fragmentados, pueden aludir a descartes cuando dan muestras de que ya no tienen potencial de uso, mientras que, si son parte de otros materiales, los consideramos piezas o elementos de la forma a la que se reconoce que pertenecieron. Algunos de los objetos aquí presentados funcionaron como colgantes o ítems almacenables, y posiblemente no fueron desechados, sino archivados, sea por su particular significación como por la facilidad que reviste su disposición en cualquier lugar o contenedor ${ }^{23}$. Por su parte, aquellos que fueron descartados de forma intencional pudieron permanecer in situ, dado su fácil ocultamiento y su resistencia a las prácticas de limpieza de lugares durante el barrido o el traslado a basurales. Aunque también es posible encontrarlos aun cuando los demás materiales con los que se asociaban fueran removidos.

Consideramos al artefacto como unidad de análisis, donde cada clase específica es representativa per se de su tipo, independientemente de su número y de las variaciones que presente. Al mismo tiempo, entendemos que, en cuanto a su materialidad, es producto de la objetivación histórica y contingente de cada grupo social, como parte de una relación constitutiva entre sujetos y objetos en un tiempo y espacios específicos ${ }^{24}$.

\footnotetext{
22 Baines, John y Málek, Jaromir. 1993. Egipto. Dioses, templos y Faraones, vol. 1, Barcelona, Folio; Lacovara, Peter. 1997. The New Kingdom Royal City, London/New York, Kegan Paul International.

${ }^{23}$ Schiffer, Michael. 1987. Formation Processes of the Archaeological Record.

${ }^{24}$ Meskell, Lynn. 2004. Object Worlds in Ancient Egypt: Material Biographies Past and Present, Oxford, Berg Publishers; Miller, Daniel. 2005. "Materiality. An Introduction”, en Daniel Miller (Ed.), Materiality, Durham/London, Duke University Press, pp. 1-50; Tilley, Christopher. 2008. "Phenomenological Approaches to Landscape Archaeology", en Bruno David \& Julian Thomas (Eds.), Handbook of Landscape Archaeology, California, Left Coast Press, pp. 271-276.
} 
Las escalas espaciales utilizadas, yendo desde lo macro a lo micro, comprenden el interior de una ciudad, una necrópolis, recintos habitacionales o tumbas. En tanto, las escalas temporales remiten a ítems que responden a un momento específico, atraviesan una generación (penicontemporáneos), o se transmiten en sucesivo a la manera de tiempo transgresivo.

\section{Baja Mesopotamia, actual Irak (2110-2003 a.C.)}

Para el estudio de las dinámicas estatales en la Baja Mesopotamia durante la Tercera Dinastía de Ur, se dispone de gran cantidad y diversidad de tablillas administrativas y de sellos cilindros, los cuales, en la actualidad, se encuentran conservados en distintos museos de Europa y Estados Unidos ${ }^{25}$.

En cuanto a la tipología documental, aunque se plantee una serie de divergencias en el interior de dicho género discursivo, los textos administrativos se caracterizan por la heterogeneidad de los archivos debido a su contenido semántico y su estructura sintáctica ${ }^{26}$. Los sitios arqueológicos donde la documentación administrativa es más abundante para este período son Ĝirsu (actual Tellōh), Puzriš-Dagān (actual Tell Drēhim) y Umma (actual Tell Ǧoḩa), los cuales funcionaron como capitales regionales y centros acaparadores de bienes. Las tablillas $\mathrm{p}$ i s a $\hat{\mathrm{g}}$ - $\mathrm{du} \mathrm{b}-\mathrm{b} \mathrm{a}^{27}$ y los sellos cilindros eran artefactos vinculados a la burocracia estatal $^{28}$.

Las tablillas p is a $\hat{\mathrm{g}}$ - $\mathrm{d}$ u b - b a , cuyo nombre traducido como "cesta de tablilla" o "tablilla contenedora" podía registrarse en el íncipit del documento, designa a un conjunto de textos que eran colocados en la parte superior de un recipiente contenedor (e.g. una canasta) a modo de etiqueta, siendo una de sus características sus dimensiones reducidas (e.g. OIP 121 539/A 3146: 4,1 cm × 3,5 cm × 2,0 cm; KM 89078/Pisan-dub-ba Texts, Diss., 183 37: 4,9 cm ×3,8 cm

\footnotetext{
${ }^{25}$ Molina, Manuel (Ed.). 2002-. Base de Datos de Textos Neosumerios. Recuperado de: http://bdts.filol.csic.es

${ }^{26}$ Steinkeller, Piotr. 2003. "Archival Practices in Babylonia in the Third Millenium", en Maria Brosius (Ed.), Ancient Archives and Archival Traditions: Concepts of Record-Keeping in the Ancient World, Oxford, Oxford University Press, pp. 37-58; Steinkeller, Piotr. 2004. "The Function of Written Documentation in the Administrative Praxis of Early Babylonia", en Michael Hudson \& Cornelia Wunsch (Eds.), Creating Economic Order, Record-Keeping, Standardization, and the Development of Accounting in the Ancient Near East, International Scholars Conference on Ancient Near Eastern Economies 4, Bethesda, CDL, pp. 64-88; Molina, Manuel. 2008. "The Corpus of Neo-Sumerian Tablets: an Overview", en Steven J. Garfinkle \& John Cale Johnson (Eds.), The Growth of an Early State in Mesopotamia: Studies in Ur III Administration. Proceedings of the First and Second Ur III Workshops at the 49 ${ }^{\text {th }}$ and $51^{\text {st }}$ Rencontre Assyriologique Internationale, London July 10, 2003 and Chicago July 19, 2005 (Biblioteca del Próximo Oriente Antiguo 5), Madrid, Consejo Superior de Investigaciones Científicas, pp. 19-53; Molina, Manuel. 2016. "Archives and Bookkeeping in Southern Mesopotamia during the Ur III Period", en Comptabilités, vol. 8, Lille. Recuperado de: http://journals.openedition.org/comptabilites/1980

${ }^{27}$ Nelson, Richard. 1976. Pisan-dub-ba Texts from the Sumerian Ur III Dynasty, University of Minnesota, Tesis para optar por al grado de Doctor.

28 Tsouparopoulou, Christina. 2013. "Reflections on Paratextual Markers and Graphic Devices in Ur III Administrative Documents”, en Textual Cultures, Vol. 8, № 2, Buffalo, pp. 1-14.
} 
$\times 2,2 \mathrm{~cm})$. De un total de $\mathrm{N}=754$ disponibles, alrededor del $60 \%(n=444)$, corresponden a Ĝirsu, mientras que un $20 \%$ ( $n=156)$ a Umma, quedando el 20\% restante distribuido entre otros sitios. Por ejemplo, la tablilla KM 89075 (MVN 9 165/ Pisan-dub-ba Texts, Diss., 351 194) (Figura 1), registra una serie de entrega de ovejas (en sumerio, $\mathrm{u} d \mathrm{u}$ ) en la ciudad de Ĝirsu durante el quinto año de reinado de Šū-Su'en:

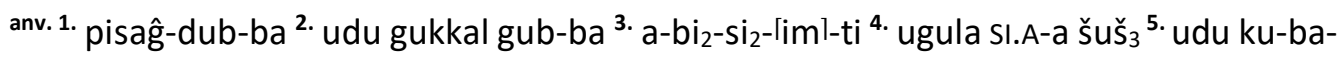
tum nin ${ }^{6}$. ugula i-zu-a-ri ${ }_{2}$-ik šušs ${ }_{3}^{7 .}$ udu e $e_{2}$-gal ${ }^{8 .}$ ugula na-wi-ir-DINGIR ${ }^{\text {rev. } 1 .}$ nam-ha-ni Iš ${ }^{2}$.

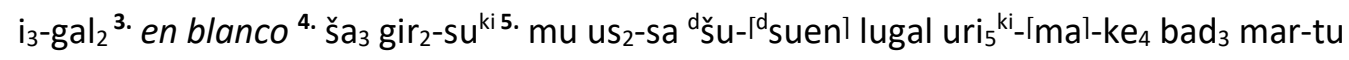
6. mu-ri-iq-ti-id-ni-im mu-du

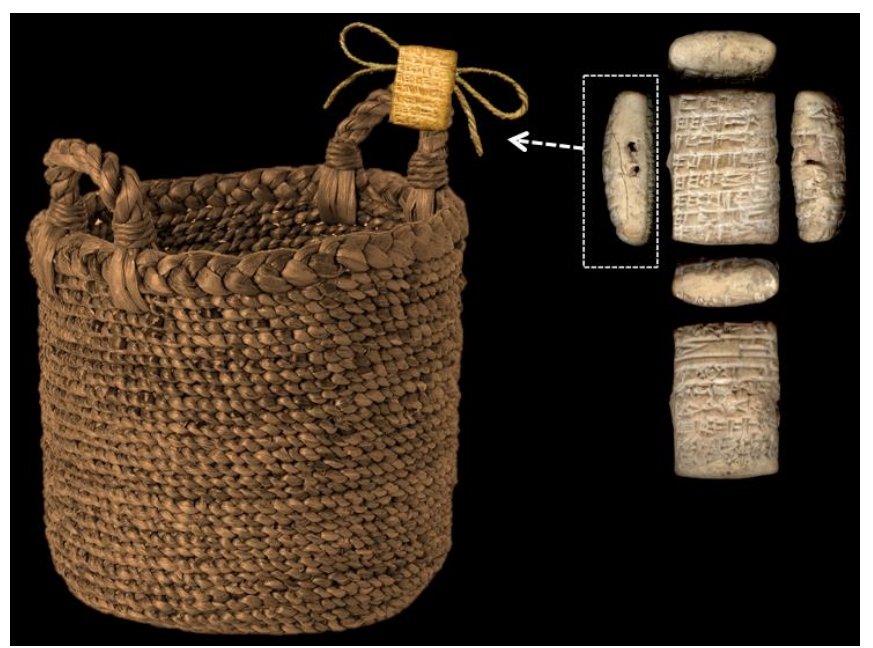

Figura 1. Tablilla p i s a $\hat{\mathrm{g}}-\mathrm{d}$ u b - b a KM 89075 con unas dimensiones de $5,6 \mathrm{~cm} \times 4,1 \mathrm{~cm} \times 2,1 \mathrm{~cm}$ y una reconstrucción de su posible uso contextual

Esta clase de artefacto se asocia con las actividades de almacenamiento y distribución de bienes a nivel estatal y local. Muchas de las tablillas pudieron estar selladas, característica que da cuenta de la existencia de un doble control. Por un lado, en el objeto mismo, se detallaban las cantidades almacenadas en cada recipiente por medio de 3 a 6 líneas escritas en el anverso y en el reverso. Por otro lado, a través del sellado, un agente del Estado se encargaba de verificar que determinadas operaciones productivas o de circulación de bienes se desarrollaran de forma eficiente.

Las tablillas "etiquetas" son reconocidas en cualquier contexto de excavación por el contenido específico del texto. No obstante, en los casos en los cuales esta denominación inicial estaba ausente, pueden ser identificadas por dos orificios que tenían en uno de los extremos a través de los cuales se enlazaba en la parte superior de un recipiente. 
Los sellos cilindros también eran artefactos asociados a la administración central, a la vez que poseían valor en cuanto objetos relacionados a un portador-dueño. En muchos casos, funcionaron como bienes suntuarios y formaron parte de los ajuares mortuorios, permitiendo identificar a los receptores al integrar las ofrendas depositadas. En el uso cotidiano, fueron indicadores de prácticas administrativas, como es el control de las actividades productivas y/o comerciales. Considerando esto último y teniendo en cuenta las prácticas vinculadas con los sellos cilindros, se conservan sus improntas sobre tablillas $\mathrm{u}$ otros objetos ${ }^{29}$.

De esta manera, para referirse a los aspectos cotidianos de las prácticas burocráticas, respecto de las tablillas $\mathrm{p}$ is a $\hat{\mathrm{g}}-\mathrm{d} \mathrm{u} \mathrm{b}-\mathrm{b} \mathrm{a}$, nos detenemos en el objeto y en su contenido textual, mientras que en relación a los sellos cilindros, nos enfocamos en los aspectos performáticos o marcas "paratextuales" ${ }^{30}$ generados por el artefacto.

\section{Tell el-Ghaba, Norte de Sinaí (siglos X - VII a.C.)}

A pesar del escaso cuidado en el registro de la proveniencia de los hallazgos pequeños en el área, se tienen algunas excepciones en las actuales excavaciones en la zona del Delta. Tal es el caso de las casas excavadas en Tell Retaba, Tell el-Ashmunein y Sais, dando prioridad a la reconstrucción de la vida cotidiana de los habitantes de los asentamientos ${ }^{31}$.

Entre los años 1995-1999 y 2010, la Misión Arqueológica Argentina realizó tareas de excavación en Tell el-Ghaba (figura 2), un asentamiento urbano que tuvo una ocupación entre comienzos del Tercer Periodo Intermedio y el periodo Saíta Temprano (mediados del siglo $\mathrm{X}$ a.C. hasta fines del siglo VII a.C. $)^{32}$. Durante estas investigaciones, fueron descubiertas diversas estructuras arquitectónicas en diferentes áreas del sitio (I, II, VI y VIII) ${ }^{33}$.

\footnotetext{
${ }^{29}$ Ibídem, pp. 10-11.

30 Ibídem, p. 10.

${ }^{31}$ Bennett, James Edward. 2019. The Archaeology of Egypt in the Third Intermediate Period, Cambridge, Cambridge University Press; Rzepka, Sławomir, Anna Wodzińska, Claire Malleson, Jozef Hudec, Łukasz Jarmużek, Krzysztof Misiewicz, Wiesław Małkowski \& Miron Bogacki. 2011. "New Kingdom and the Third Intermediate Period in Tell elRetaba: Results of the Polish-Slovak Archaeological Mission, Seasons 2009-2010", en Ägypten und Levante/Egypt and the Levant, vol. 21, Wien, pp. 129-184; Wilson, Penelope \& Louise Bertini. 2011. Sais I: The Ramesside-Third Intermediate Period at Kom Rebwa, London, Egypt Exploration Society.

${ }^{32}$ Basílico, Susana \& Lupo, Silvia (Eds.). 2006. Tell el-Ghaba, norte de Sinaí, Egipto. Alimentación, producción e intercambio, Buenos Aires, Dunken; Lupo, Silvia (Ed.). 2015. Tell el-Ghaba III. A Third Intermediate-early Saite Period Ssite in the Egyptian Eastern Delta. Excavations 1995-1999 and 2010 in areas I, II, VI and VIII (BAR International Series 2756), Oxford, Archaeopress.

${ }^{33}$ Lupo, Silvia (Ed.). 2015. Tell el-Ghaba III; Lupo, Silvia, Eduardo Crivelli Montero, Claudia Kohen \& Eva A. Calomino. 2017. "Building B, a Domestic Construction at Tell el-Ghaba, North Sinai", en Rosati, Gloria y Guidotti, Cristina (Eds.), Proceedings of the XI International Congress of Egyptologists. Florence Egyptian MuseumFlorence, 23-30 August 2015 (Archaeopress Egyptology 19), Oxford, Archaeopress, pp. 351-356.
} 


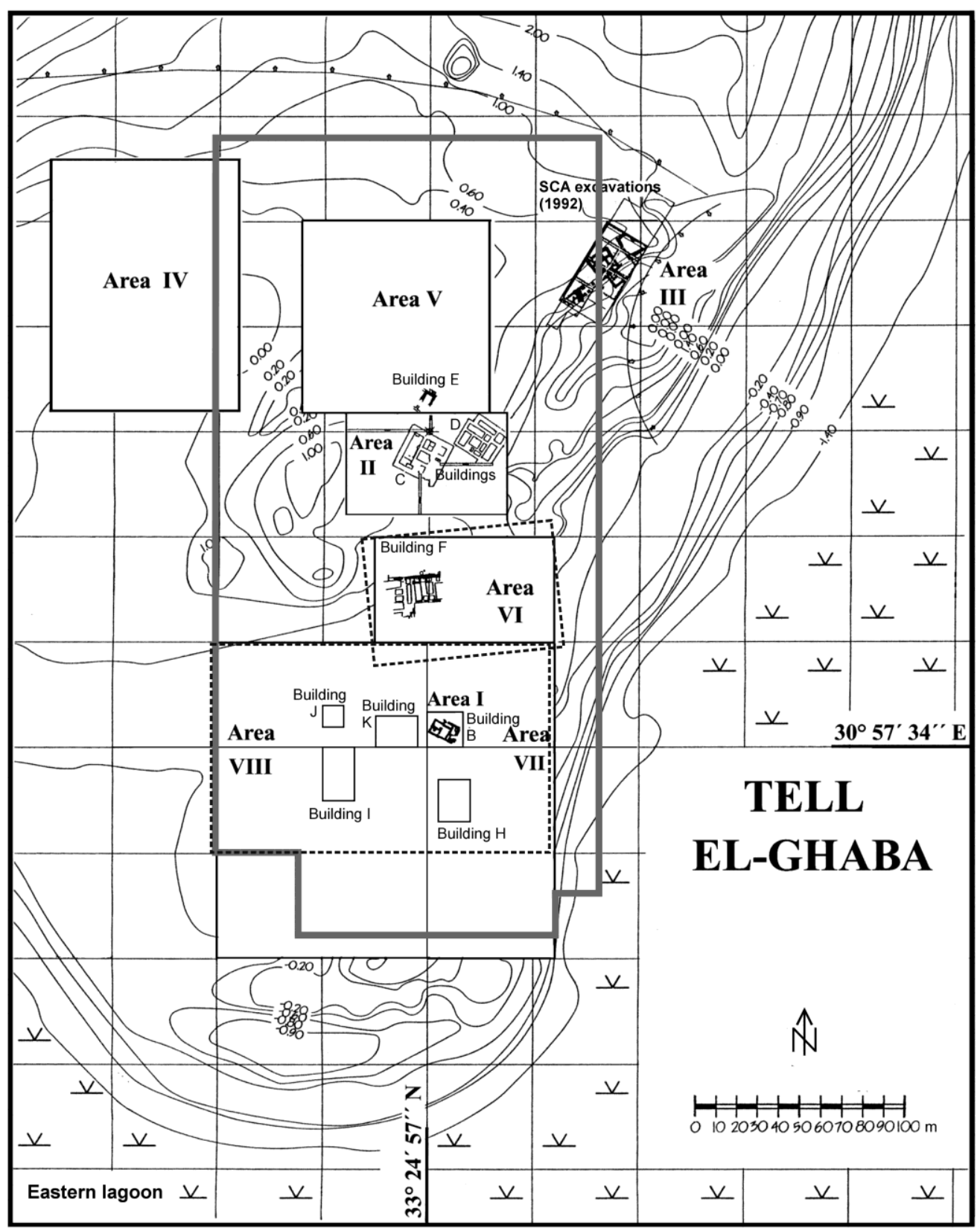

Figura 2. Plano de Tell el-Ghaba con las áreas de excavación y los edificios excavados.

El estudio de los materiales recuperados en tales estructuras sugiere que las actividades dominantes fueron domésticas, tales como la preparación y consumo de comida, el almacenamiento de granos, líquidos, entre otras posibilidades. Dicha interpretación se ve reforzada por los datos obtenidos a partir de los análisis de los conjuntos cerámicos y restos 
faunísticos ${ }^{34}$. Sin embargo, las actividades que habrían tenido lugar en cada una de estas unidades domésticas no fueron exclusivamente de ese tipo. Por ejemplo, para el caso del Edificio $B$, se ha propuesto que las actividades habrían sido múltiples, con un uso integrado de las diferentes habitaciones para desplegar un conjunto de prácticas cotidianas ${ }^{35}$.

Las investigaciones en este sitio requieren el análisis de diversos materiales, como cerámica, small finds, restos faunísticos, líticos, entre otros, a los efectos de reconocer las actividades llevadas a cabo por sus habitantes. Los pequeños hallazgos fueron recuperados en excavaciones sistemáticas, en contextos bien definidos, registrados y analizados bajo condiciones de laboratorio controladas que permiten ahondar en el conocimiento de las relaciones e interacción entre las personas y los objetos ${ }^{36}$.

Con el fin de conocer las actividades que realizaban los habitantes de las unidades domésticas a nivel local y comunal y de sus creencias, se llevó a cabo una categorización específica para evitar establecer funcionalidades a priori. Entonces, a grandes rasgos, estos conjuntos se han clasificado por: a) características: morfología, materia prima, dimensiones, color, técnicas de manufactura del objeto, rasgos, condición, estado de conservación, alteraciones naturales, huellas de uso, composición temática/decorativa, técnica de la composición, forma geométrica; $b$ ) contextos: área, cuadrícula, locus, estructura.

El catálogo de small finds $(\mathrm{N}=964)^{37}$ está conformado no solo por artefactos de reducidas dimensiones, sino también por una cantidad de "hallazgos especiales", como pesos de telar,

\footnotetext{
${ }^{34}$ Basílico, Susana y Lupo, Silvia (Eds.). 2006. Tell el-Ghaba, norte de Sinaí; Cione, Alberto. 2006. "Fishes from Tell elGhaba", en Perla Fuscaldo (Ed.), Tell el-Ghaba II. A Saite settlement in North Sinai, Egypt (Argentine Archaeological Mission 1995-2004). Vol. II: Studies (Colección Estudios 5), Buenos Aires, Instituto Multidisciplinario de Historia y Ciencias Humanas-Consejo Nacional de Investigaciones Científicas y Técnicas, pp. 102-136; Lupo, Silvia (Ed.). 2015. Tell el-Ghaba III.

35 Lupo, Silvia; Calomino, Eva y Scaro, Agustina. 2019. "El Edificio B de Tell el-Ghaba como un caso de estudio para repensar la dinámica de la unidad doméstica en el Antiguo Egipto", en Trabajos de Egiptología, Vol. 10, Tenerife, pp. 201-216.

36 Calomino, Eva. 2019. "Los small finds de Tell el-Ghaba (Norte de Sinaí, Egipto). Una propuesta teóricometodológica para analizar los hallazgos especiales en contextos domésticos entre los siglos X y VII a.C.", en Actas de la I Jornada en Investigación y Docencia sobre el Cercano Oriente Antiguo, 2 y 3 de noviembre de 2017, FaHCE, UNLP, La Plata, Argentina, La Plata, Facultad de Humanidades y Ciencias de la Educación, Universidad Nacional de La Plata, pp. 1-10. Recuperado de: http://www.memoria.fahce.unlp.edu.ar/trab_eventos/ev.13178/ev.13178.pdf; Calomino, Eva. En prensa. "Note per lo studio di 'risultati speciali' in contesti domestici. Small finds di Tell el-Ghaba (Nord Sinai, Egitto)", en VRBS. Studi sulla romanità antica e tardoantica, Vol. 3; Calomino, Eva; Scaro, Agustina y Lupo, Silvia 2017. "Contextos, actividades y funciones en una estructura privada de Tell el-Ghaba (Norte de Sinaí, Egipto): el Edificio B", en Laura Burgos Bernal, Antonio Pérez Largacha \& Inmaculada Vivas Sainz (Coords.), Congreso Ibérico de Egiptología (Colección Estudios 157), Cuenca, Universidad de Castilla/La Mancha, p. 157; Lupo, Silvia, Eduardo Crivelli Montero, Claudia Kohen \& Eva A. Calomino. 2017. "Building B, a Domestic Construction at Tell elGhaba, North Sinai", 351-356.

${ }^{37}$ Fuscaldo, Perla (Ed.). 2005. Tell el-Ghaba I. A Saite Settlement in North Sinai, Egypt (Argentine Archaeological Mission 1995- 2004). Vol I. The Catalogue (Colección Estudios 5), Buenos Aires, CONICET.

Fuscaldo, Perla (Ed.). 2006. Tell el-Ghaba II. A Saite Settlement in North Sinai, Egypt (Argentine Archaeological Mission 1995- 2004). Vol II. Studies (Colección Estudios 5), Buenos Aires, CONICET.
} 
pesos de red, fragmentos de rocas y materias primas, instrumentos líticos, tope de puerta, betún, fragmentos de escoria de metal y de fayenza. Dentro de estos, la base de datos de pequeños objetos pertenecientes a Tell el-Ghaba consta de: 66 wdjat, 43 figurinas/fragmentos de estatuas y amuletos, 13 escarabajos y escaraboides, 5 pendientes o colgantes, 3 anillos, 193 cuentas, 4 plaquetas, 2 sellos, 8 anzuelos, 35 piezas de juego y una espátula.

De forma general, es posible establecer que, en las unidades domésticas, habrían tenido lugar una diversidad de actividades que incluían el uso de una gran variedad de small finds categoría que incluye pequeños objetos y otros "hallazgos especiales"- y de pequeños objetos. Estos habrían sido utilizados principalmente como adornos corporales y del mobiliario y como amuletos. Figurinas Bes, wdjats, una estatuilla Hathor y otros reflejan las creencias trdicionales egipcias de los habitantes de este sitio de la frontera oriental del Estado egipcio en la ruta que une Egipto y el Levante, el llamado Camino de Horus.

\section{Tell el-Amarna, Egipto (1350-1335 a.C.)}

La muestra consta de un conjunto de evidencia proveniente de distintas áreas del sitio Tell elAmarna y es una selección de hallazgos ( $\mathrm{N}=2385)$ publicados en la Small Finds Database del Amarna Project (2017). La misma incluye información detallada de objetos recuperados por la EES entre los años 1921 y 1936 que fue compilada de las publicaciones de City of Akhetaten I $^{38}$, $11^{39}$ y $\| I^{40}$.

El lugar de hallazgo es la ciudad de Akhetatón, ubicada en la actual Tell el-Amarna, la que a su vez da nombre al período y funcionó como centro político y religioso durante el gobierno de Akhenatón (1355-1338 a.C.). La organización territorial de este paisaje fue funcional al establecimiento de la realeza y la élite, como el nuevo polo religioso dedicado al dios Atón, posicionando a este último como deidad estatal principal, en detrimento del dios Amón, entre otras cuestiones. La religión oficial de Amarna destaca porque la conexión con el disco solar pasó a ser privilegio del faraón y de su familia, por ser estos los ejecutantes del culto y únicos intermediarios con la deidad.

La evidencia arqueológica de Tell el- Amarna se encuentra muy sesgada debido a que el Estado reformador atoniano planificó el trazado de la ciudad para trascender en el tiempo. Luego, fue profundamente alterada con la restitución del dios Amón al culto oficial, practicando

\footnotetext{
Lupo, Silvia (Ed.). 2015. Tell el-Ghaba III. A Third Intermediate-early Saite Period in the Egyptian Eastern Delta. Excavations 1995-1999 and 2010 in areas I, II, VI and VIII (BAR International Series 2756), Oxford, Archaeopress.

38 Peet, Thomas y Woolley, Leonard. 1923. The City of Akhenaten, I: Excavations of 1921 and 1922 at el-'Amarna, London, Egypt Exploration Society.

${ }^{39}$ Frankfort, Henri; Fairman, Herbert y Pendlebury, John. 1933. The City of Akhenaten, Part 2, The North Suburb and the Desert Altars: the Excavations at Tell el Amarna during the Season 1926-1932, London, Egypt Exploration Society. ${ }^{40}$ Pendlebury, John. 1951. The City of Akhenaten: Part III: The Central City and the Official Quarters. The Excavations at Tell-El-Amarna during the Seasons 1926-1927 and 1931-1936, London, Egypt Exploration Society.
} 
damnatio memoriae, desmantelando estructuras monumentales $\mathrm{y}$, finalmente, con el abandono del lugar. A esto, se sumaron otros agentes y procesos disturbadores, planteando la destrucción de los contextos de uso de los objetos. Por su parte, las excavaciones arqueológicas, iniciadas por Petrie a finales del siglo XIX y seguidas por el Deutsche Orient-Gesellschaft (DOG) y la Egyptian Exploration Society (EES), más allá de que estuvieron dirigidas a despejar y registrar estructuras arquitectónicas tanto oficiales (templos y palacios) como domésticas, continuaron afectando los contextos de depositación del sitio. Actualmente, investigaciones sistemáticas son realizadas por el Amarna Project ${ }^{41}$.

Asimismo, durante las intervenciones más tempranas las piezas recuperadas fueron las más vistosas. Mientras que otros objetos habrían sido ignorados y descartados junto con los sedimentos removidos durante la excavación. En ambos casos, debieron resultar favorecidos aquellos materiales merecedores de ser expuestos o conservados en museos, mientras que otros, como joyas e incluso recipientes de baja calidad, fueron vendidos o donados, circulando ya despojados de sus contextos estratigráficos ${ }^{42}$.

Los objetos aquí analizados, por su forma, decoraciones o inscripciones, poseen simbolismos y asociaciones con algún tipo de práctica religiosa, ya sea popular (cultos tradicionales) o estatal (Atón y realeza). Se los identifica, por sus funciones y usos, como joyas (aros y anillos), amuletos, moldes de arcilla de figuras y jeroglíficos (e.g. anj y wdjats), ostracas, vasijas y objetos votivos, entre los que encontramos pequeñas estatuillas. Bes, Tawret y Hathor aparecen formando parte de las deidades asociadas con la religión tradicional, representadas bajo símbolos o a través de sus figuras moldeadas. Dada su conexión con la fertilidad y los partos habrían sido usadas como amuletos ${ }^{43}$. Este es el tipo de objeto más abundante y pertenece a la categoría joyería -y sus moldes-, los cuales fueron hallados generalmente divisos. Asimismo, encontramos pequeños fragmentos de estructuras arquitectónicas, relieves y estatuas de dimensiones reducidas.

Los hallazgos religiosos aparecen en diferentes cantidades y áreas de la ciudad, siendo el Pueblo de Trabajadores, la Ciudad Central, la Ciudad Principal, el Marú Atón, la Ciudad Norte, el Suburbio Norte y el Palacio Norte las incluidas en este trabajo. Las concentraciones de objetos asociados con la religión oficial aparecen en áreas conectadas con el Estado y la realeza por albergar edificios oficiales (como templos y palacios), aunque también aparecen en otras áreas. Por su parte, la dispersión de los objetos asociados con la religión tradicional, que representan un $75 \%$ del total de hallazgos religiosos, se presenta en todas las áreas, incluyendo las oficiales (figura 3).

\footnotetext{
${ }^{41}$ Stevens, Anna. 2015. Archaeology of Amarna, Oxford, Oxford Handbooks Online.

${ }^{42}$ Stevens, Anna. 2015. Archaeology of Amarna, p. 8.

${ }^{43}$ Pinch, Geraldine. 1994. Magic in Ancient Egypt, London, British Museum Press, p. 106.
} 

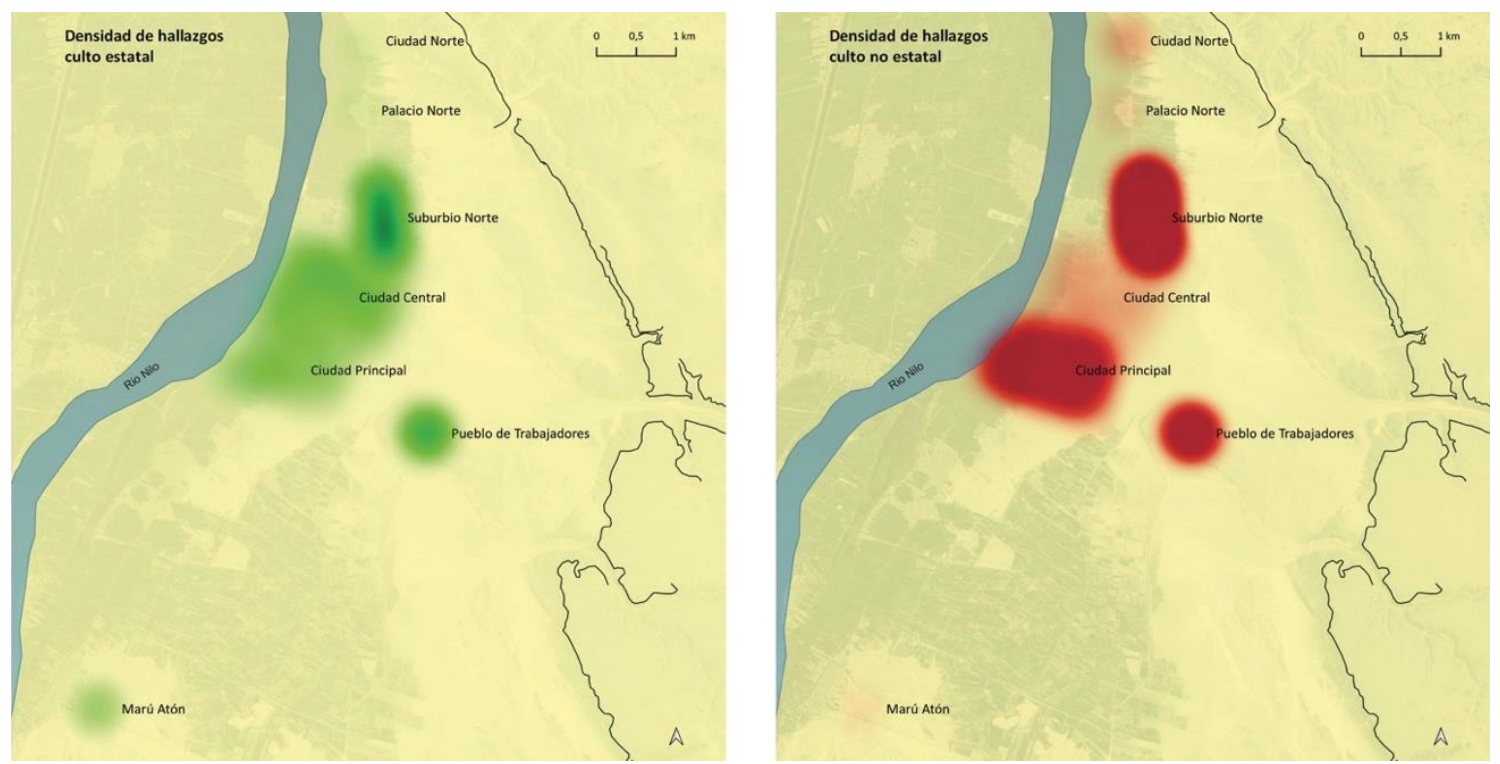

Figura 3. Mapas de calor de Amarna con densidad de hallazgos de culto estatal y no estatal

La evidencia textual disponible, como las cartas a los difuntos ${ }^{44}$, permite considerar que los diferentes grados de jerarquía y cercanía con el poder central determinaron diferentes modalidades de acceso a la religión oficial ${ }^{45}$. Las diversas líneas de evidencia, sumadas a la dispersión de los hallazgos en Tell el-Amarna, habilitan la posibilidad de que las prácticas religiosas asociadas con el culto estatal y los cultos populares y/o tradicionales atravesaran a toda la población y, por ende, fueran practicados tanto por la élite como por la no-élite ${ }^{46}$.

Mientras el culto estatal tenía lugar en espacios planeados desde el Estado para tal fin, las prácticas religiosas cotidianas y ligadas con la religión tradicional se realizaban utilizando lugares que no se correspondían con actividades particulares ${ }^{47}$. Sin embargo, esto no necesariamente significa que los dos tipos de culto se practicaran de forma separada por cada categoría de habitante (la élite o la no-élite) o tipo de espacio (oficial o cotidiano). De hecho, la distribución de los objetos da cuenta de un escenario diverso y poco escindido, donde la evidencia de prácticas religiosas aparece incluso donde no habría espacios definidos para su desarrollo ${ }^{48}$.

\footnotetext{
${ }^{44}$ Baines, John. 2001. "Egyptian Letters of the New Kingdom as Evidence for Religious Practice", en Journal of Ancient Near Eastern Religions, Vol. 1, №1, Leiden, pp. 1-31.

${ }^{45}$ Bickel, Susanne. 2003. "'Ich spreche ständig zu Aton...': zur Mensch-Gott-Beziehung in der Amarna Religion”, en Journal of Ancient Near Eastern Religions, Vol. 3, Leinden, pp. 23-45.

${ }^{46}$ Stevens, Anna. 2006. Private Religion at Amarna. The Material Evidence, Oxford, Archeopress.

47 Ídem.

48 lamarino, María Laura. 2018. "Evidencia material y culto a deidades tradicionales en Amarna", en Antonio Brancaglion \& Gisela Chapot (Eds.), Semana de Egiptologia do Museu Nacional (SEMNA) (Estudos de Egiptologia V), Rio de Janeiro, Seshat-Laboratório de Egiptologia do Museu Nacional Editora e Klínē, pp. 106-121.
} 
Probablemente esto se deba a que los diferentes cultos implicaron distintos momentos y lugares en los cuales entablar la relación entre las personas con las divinidades, como ser con las asociadas con la fertilidad en una conexión de carácter más individual, mientras que otros estuvieron mediados por la familia real y tuvieron un amplio alcance. No resultaron necesariamente contradictorios sino formando parte de una dinámica social compleja ${ }^{49}$, dada la alternancia de espacios, ocasiones y manifestaciones personales o colectivas.

Asimismo, la evidencia dispersa de objetos relacionados con todo tipo de culto y la mayor abundancia de los ligados a la práctica religiosa tradicional pudo deberse a diferentes motivos. Uno fue el proceso mediante el cual se buscó eliminar los rastros de Akhenatón una vez restituido el culto a Amón, lo que pudo generar un desbalance en la cantidad de evidencia ligada a la religión ahora suprimida. Otra causa puede relacionarse con el hecho de que el asentamiento continuara habitado luego de desaparecido Akhenatón ${ }^{50}$, quien fuera el principal impulsor de la práctica atoniana.

\section{Tebas Occidental, Egipto (1550-1307 a.C.)}

Tebas Occidental es un espacio sacralizado dedicado en mayor medida a la depositación de momias y a la realización de rituales funerarios. Entre las $\mathrm{N}=906$ tumbas de la élite tebana que se contabilizan en la actualidad ${ }^{51}$, fueron valoradas especialmente aquellas en las que todavía se preservaban momias, ajuares y registros parietales. En la mayoría de los casos, los contextos materiales se encuentran muy perturbados, motivando a que su estudio sea desestimado y se privilegie la recuperación de registros epigráficos ${ }^{52}$.

Una característica compartida por las tumbas tebanas es la compleja historia postocupacional en la que se vieron involucradas luego de su abandono como lugar de entierro. Entre los varios deterioros que registran, pueden mencionarse los saqueos (desde la antigüedad hasta tiempos recientes), los incendios (entre fines del siglo XIX y principios del XX), su ocupación como vivienda o parte de estas (desde comienzos del siglo XX hasta la década de los

\footnotetext{
${ }^{49}$ Bussmann, Richard. 2011. "Seals and Seal Impressions from Hierakonpolis", en Egyptian Archaeology. The Bulletin of the Egypt Exploration Society, Vol. 38, London, pp. 17-19; Dieleman, Jacco. The materiality of textual amulets in ancient Egypt, en Boschung, Dietrich; Bremmer, Jan. (Eds.), The Materiality of Magic (Morphomata 20), Paderborn, Wilhelm Fink, pp. 23-58

50 Shaw, lan. 1984. "Ring Bezels at el-Amarna", en Barry Kemp (Ed.), Amarna reports I, London. Egypt Exploration Society, pp. 124-132.

${ }^{51}$ Manzi, Liliana. 2018. "Personas y lugares en el paisaje de Tebas Occidental, Egipto", pp. 90-105.

52 Gardiner, Alan \& Arthur E. P. Brome Weigall. 1913. A Topographical Catalogue of the Private Tombs of Thebes, London, Bernard Quaritch; Porter, Bertha \& Rosalind Moss. 1970. Topographical Bibliography of Ancient Egyptian Hieroglyphic Texts, Reliefs, and Paintings. I. The Theban Necropolis, Part 1. Private Tombs, Oxford, Griffith Institute/Ashmolean Museum.
} 
noventa) y visitas sin ninguna clase de control (desde fines del siglo XVIII hasta la década de los ochenta), entre algunas otras intervenciones ${ }^{53}$.

El estudio de la TT49, Tumba de Neferhotep (reinado de Ay -1327-1323 a.C.-), puede ser tomado como un caso de referencia ${ }^{54}$ por las semejanzas que guarda con otras tumbas de la necrópolis en cuanto a las perturbaciones que presentan ${ }^{55}$. Las estratigrafías verticales de las paredes de este monumento permitieron reconocer procesos de construcción, mantenimiento, ciclado de escenas por cambios ideológicos -luego de la proscripción religiosa post-Amarna-y su reciclado -con la apertura de un sepulcro para un propietario distinto del original- ${ }^{56}$. Asimismo, el registro artefactual y bioarqueológico los daños ocurridos desde su abandono y que llevaron a la destrucción, remoción y mezcla de esta evidencia ${ }^{57}$.

Su estudio considera tanto materiales in situ como los que se encontraban redepositados ${ }^{58}$. Así se reconocieron fragmentos de carbonillas $(n=4)$ y restos de fritas $(n=11)$ utilizados en la decoración parietal y que pueden ser considerados desechos de facto ${ }^{59}$. Además, se registraron fragmentos del equipo funerario, tales como estatuillas momiformes -shabtis- ( $n=7)$, amuletos -Isis- $(n=2)$, escarabajos $(n=2)$, fragmentos de textiles -vendas con distintos grosores de hilado( $n=107)$, vegetales - guirnaldas florales- $(n=42)$, cuentas y canutillos de cerámica, fayenza y roca ( $n=417)$, además de trozos de madera de sarcófagos $(n=68)$ y de cartonnage $(n=14)$. Y, como material intrusivo, se registra, entre otros, un cono funerario fracturado que fue parte de la decoración de las fachadas (figura 4 y tabla 1 ).

\footnotetext{
53 Manzi, Liliana. 2009-2010. "Intervenciones y registro de daños en la tumba de Neferhotep, la colina de el-Khokha, Tebas occidental, Egipto", en Avances. Revista del Área Artes, no. 16, Córdoba, pp. 187-201; Pereyra, María Violeta, Manzi, Liliana y Broitman, Livia. 2013. "La Tumba Tebana 49 y su propietario, en el paisaje sacralizado del occidente tebano, Egipto", en Arqueología, Vol.19, Buenos Aires pp. 103-123; Strudwick, Nigely Strudwick, Helen. 1999. Thebes in Egypt: A Guide to the Tombs and Temples of Ancient Luxor, London, British Museum Press; Simpson, Caroline. 2003. "Modern Qurna-Pieces of an Historical Jigsaw", pp. 244-249.

${ }^{54}$ Pereyra, María Violeta, Maria Giorgia Di Antonio, Maria Violeta Carniel \& Oliva Menozzi. 2015. "Il complesso funerario di Neferhotep (Luxor): una lunga storia di 'riutilizzi'. Progetto, metodologie, tecnologie, scavo e protocolli archeometrici integrati", en Frankfurter elektronische Rundschau zur Altertumskunde, vol. 28, Flensburg, pp. 17-62. Recuperado de: http://www.fera-journal.eu/index.php/ojs-fera/article/view/156

55 Strudwick, Nigel \& John Taylor (Eds.). 2003. Present and Past in the Theban Necropolis, London, The British Museum Press.

${ }^{56}$ Manzi, Liliana. 2009-2010. "Intervenciones y registro de daños en la tumba de Neferhotep, la colina de el-Khokha, Tebas occidental, Egipto”, pp. 187-201.

57 Pereyra, María Violeta, Manzi, Liliana y Broitman, Livia. 2013. "La Tumba Tebana 49 y su propietario, en el paisaje sacralizado del occidente tebano, Egipto", pp. 103-123.

58 Manzi, Liliana y Sánchez, Julián. 2007. "Bloques de distintas procedencias alojados en la tumba de Neferhotep (TT49), el-Khokha (Tebas occidental, Egipto)", en Actas del I Congresso Internacional da Sociedade de Arqueología Brasileira / XIV Congresso da SAB/III Encontro do IPHAN e Arqueólogos, Florianópolis, Sociedade de Arqueología Brasileira, pp. 95-107; Pereyra, María Violeta, Cerezo, María Eugenia y Fresquet, Inés 2007. "El aporte de los registros fragmentarios para la reconstrucción de la historia de una tumba egipcia", en Actas del I Congresso Internacional da Sociedade de Arqueología Brasileira/XIV Congresso da SAB/III Encontro do IPHAN e Arqueólogos, Florianópolis, Sociedade de Arqueología Brasileira, pp. 1-15.

${ }^{59}$ Schiffer, Michael. 1987. Formation Processes of the Archaeological Record.
} 


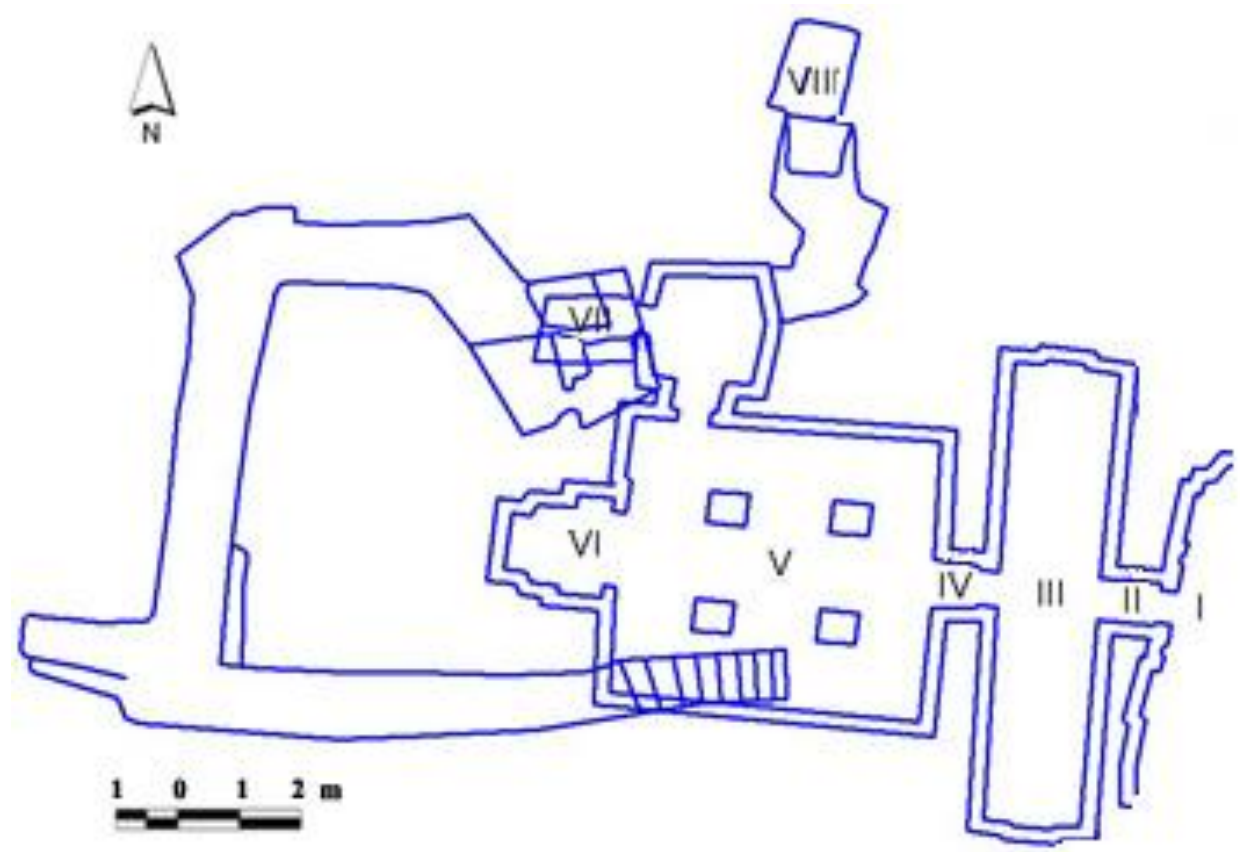

Figura 4. Sectores: I patio (exterior), II primer pasaje, III vestíbulo, IV segundo pasaje, V-VI capilla de culto y del ka, VII sepulcro principal y VIII sepulcro usurpador

Tabla 1. Presencia de objetos por sectores

\begin{tabular}{|c|c|c|c|c|}
\hline Materiales/sectores & sector III & sectores V-VI & sector VII & sector VIII \\
\hline Amuletos & & $\mathrm{X}$ & & \\
\hline Carbonillas & $\mathrm{X}$ & & & \\
\hline Cartonaje & & $\mathrm{X}$ & & $\mathrm{X}$ \\
\hline Cuentas/canutillos & & $\mathrm{X}$ & & $\mathrm{X}$ \\
\hline Escarabajos & & $\mathrm{X}$ & & \\
\hline Fritas & $\mathrm{X}$ & $\mathrm{X}$ & & $\mathrm{X}$ \\
\hline Maderas & & $\mathrm{X}$ & $\mathrm{X}$ & $\mathrm{X}$ \\
\hline Shabtis & & $\mathrm{X}$ & & \\
\hline Textiles & & $\mathrm{X}$ & & $\mathrm{X}$ \\
\hline Vegetales & & & & $\mathrm{X}$ \\
\hline
\end{tabular}

Fuente: elaboración propia.

En el caso de la TT49, al igual que en otros monumentos de la necrópolis, la información proporcionada por los pequeños objetos da cuenta de por lo menos tres cuestiones. Una de ellas, relacionada con los procesos de decoración de hipogeos, luego de alisadas las paredes, entre las etapas iniciales del programa decorativo, se procedía a delinear textos y escenas con carbonillas y en momentos más avanzados, se continuaba con el pintado mediante el uso de 
fritas y otros pigmentos ${ }^{60}$. Otra cuestión apunta al equipamiento mortuorio. La última refiere a la presencia del cono funerario que responde a una antigua costumbre de visitar lugares sacralizados, donde las personas dejaban un recordatorio de su paso y una plegaria al difunto ${ }^{61}$.

\section{¿Tamaños reducidos, grandes complejidades?}

El estudio de pequeños hallazgos permite reconocer tanto prácticas individuales como aquellas que fueron representativas de ciertos agentes sociales (la realeza, las élites y las no élites). Estas prácticas no fueron exclusivamente realizadas por determinados agentes según su grupo de pertenencia, sino que fluyeron entre unos y otros. Son expresivas del contexto histórico al que corresponde la evidencia, señalando la presencia estatal y cómo esta extiende, de forma explícita o implícita, sus mecanismos de control.

En Mesopotamia, la manufactura de sellos cilindros, empleados para sellar los documentos que referían a transacciones ejecutadas por agentes privados, y los textos administrativos que estos mismos se hacían confeccionar ponen de manifiesto el solapamiento y la tensión entre las esferas pública y privada. En muchos casos, agentes particulares, como pueden ser los comerciantes, emulaban determinadas prácticas estatales.

Los desempeños individuales son, en alguna medida, representativos del colectivo que conforma la sociedad y encarnan la adhesión voluntaria o coercitiva de muchos otros actores sociales respecto de creencias y modas particulares. Asimismo, advertimos que ciertas prácticas de carácter personal no se restringen solo al ámbito de lo cotidiano o doméstico, sino que operan dentro de los dominios públicos, los cuales, sin pretender incidir o modificarlos, exponen el apego a prácticas tradicionales. Incluso podrían dar cuenta de aspectos identitarios, en tanto factores de diferenciación o de resistencia a imposiciones. Las esferas privadas tampoco responden ni única, ni necesariamente al ámbito doméstico o estrictamente individual. Estos comportamientos no se circunscriben a un único sector social, sino que lo trascienden, en particular cuando las decisiones de orden público se dirimen en la órbita parental, asumiendo un carácter colectivo, donde lo personal no responde únicamente a lo individual.

El otorgamiento de tumbas a los nobles tebanos por parte del Estado da cuenta de su acceso por parte de individuos que pueden provenir de cualquier estamento de la élite. Ese beneficio se relaciona con su desempeño en la esfera pública y también de las negociaciones y alianzas que se despliegan desde los linajes ${ }^{62}$. Las diferentes calidades, variedades y cantidades de

\footnotetext{
60 Hartwig, Melinda. 2004. Tomb Painting and Identity in Ancient Thebes.

${ }^{61}$ Manniche, Lise. 2001. "Funerary Cones", en Donald Redford (Ed.), The Oxford Encyclopedia of Ancient Egypt I, Oxford, Oxford University Press, pp. 565-567.

62 Manzi, Liliana. 2012. "La jerarquización del espacio a través de la distribución de tumbas privadas en Tebas Occidental, Egipto", en Luís Manuel de Araújo \& José das Candeias Sales (Eds.), Novos Trabalhos de Egiptologia Ibérica, vol. 1, Lisboa, Instituto Oriental e Centro de História da Faculdade de Letras da Universidade de Lisboa, pp.
} 
hallazgos reflejan distintos grados de relación con el Estado. Por ejemplo, se espera que los más altos dignatarios presenten un número mayor de elementos en su ajuar funerario, como es el caso de los shabtis.

En los contextos domésticos, tales como los mencionados en Sinaí, los pequeños hallazgos están relacionados espacialmente con otros artefactos y estructuras -como los hornos-, cuyo estudio ha permitido delinear una variedad de actividades desplegadas entre espacios con mayor o menor privacidad. En este sentido, el uso de las habitaciones estuvo ligado a factores sociales internos, así como a la relación existente con el espacio exterior, sin excluir la idea de una planificación según ciertas expectativas, también sociales ${ }^{63}$.

En parte, la compleja interacción entre el dominio oficial y las decisiones personales queda expuesta en la práctica de cultos tradicionales durante el gobierno de Akhenatón en Amarna. Allí, los pequeños hallazgos comprenden objetos que eran utilizados tanto en asociación con prácticas estatales como no estatales, atravesando a los diferentes sectores de la población y desarrollándose de forma comunitaria y/o privada.

A excepción de alguna evidencia (e.g. restos de carbonilla y fritas de pigmentos) es muy difícil garantizar que esta se ubique en su posición original, encontrándose por defecto redepositada y, en consecuencia, sus referencias temporales y contextuales se presentan modificadas. No obstante, hay artefactos que pueden ser asociados de forma directa con sus contextos de uso, a pesar de encontrase redepositados, como aquellos materiales que fueron parte del ajuar mortuorio y se hallan en contextos funerarios. En tanto que otros aparecen en contextos diferentes de los que se espera que formaran parte originalmente. En ese caso, las posibilidades son que los objetos se manifiesten por completo descontextualizados o que posean la capacidad de participar indistintamente en diferentes ámbitos (públicos y/o privados).

\section{Conclusiones}

A través de los casos analizados, se observa que los pequeños objetos responden a distintas clases de uso a pesar de su situación de hallazgo y de que en la actualidad sean parte de colecciones. Nos centramos en sus tamaños reducidos sin ponderar la rareza de sus formas, el grado de especialización que requirieron para su elaboración o la singularidad de sus materias primas, sino porque tuvieron la facultad de perdurar a pesar de conformar registros alterados.

637-655; Manzi, Liliana. 2016. “Monumentalización e evocación en el paisaje de Tebas occidental, Egipto”, en Revista Mundo Antigo. Dossiê Egiptologia, Vol. 5, № 9, Río de Janeiro, pp. 191-205; Manzi, Liliana. 2018. “Personas y lugares en el paisaje de Tebas Occidental, Egipto", en Antonio Brancaglion \& Gisela Chapot (Eds.), Semana de Egiptologia do Museu Nacional (SEMNA) (Estudos de Egiptologia V), Rio de Janeiro, Seshat-Laboratório de Egiptologia do Museu Nacional Editora e Klínē, pp. 90-105.

${ }^{63}$ Lupo, Silvia; Calomino, Eva y Scaro, Agustina. 2019. “El Edificio B de Tell el-Ghaba...”, pp. 201-216. 
A juzgar por la evidencia recuperada y sus contextos, las prácticas personales se relacionan con los ámbitos domésticos y públicos, tanto de forma alternada como de modo simultáneo. De este modo, se observa que las prácticas y los pequeños objetos trascienden los ámbitos de uso específico y se integran tanto a los dominios públicos como privados.

Esta división es más analítica que real, dado que una clase o diseño de objetos puede desenvolverse de modo transversal. Es de esperar que elementos de uso cotidiano tengan incidencia tanto en ámbitos públicos como privados y se validen en una u otra esfera, dependiendo de las prácticas que forman parte de la cotidianidad de las personas. Creemos que el estudio de artefactos pequeños, con mayor capacidad de ser transportados y/o almacenados, es un abordaje promisorio para el reconocimiento de esferas de interacción entre lo público y lo privado, lo cotidiano y lo esporádico, y lo discursivo y su correlato material.

A partir de la interacción de las personas, se tejen palimpsestos de significación y, en consecuencia, los objetos también se inscriben en una dimensión inmaterial que trasciende las áreas de desempeño específico de las personas.

\section{Referencias citadas}

Allison, Penelope. 1999. The Archaeology of Household Activities, London, Routledge.

AA.VV. 2017. The Amarna Small Finds Database, Disponible en: https://www.amarnaproject.com/pages/recent_projects/material_culture/small_finds/database.sh tml (Consultado el 20 de diciembre de 2020)

Appadurai, Arjun. 1986. "Introduction: Commodities and the Politics of Value", en Arjun Appadurai (ed.), The Social Life of Things: Commodities in Cultural Perspective, New York, Cambridge University Press, pp. 3-63.

Assmann, Jan. 2005 (1996). Egipto. Historia de un sentido, Madrid, Abada Editores.

Bailey, Geoff. 2005. "Concepts of Time", en Renfrew, Colin \& Bahn, Paul (eds.), Archaeology: The Key Concepts, London/New York, NY, Routledge, pp. 200-203.

Bailey, Geoff. 2007. "Time Perspectives, Palimpsests and the Archaeology of Time", en Journal of Anthropological Archaeology, vol. 26, № 2, Amsterdam, pp. 198-223.

Bailey, Geoff. 2007. "Time Perspectives, Palimpsests and the Archaeology of Time", en Journal of Anthropological Archaeology, vol. 26, № 2, Amsterdam, pp. 198-223.

Baines, John \& Jaromir Málek. 1993. Egipto. Dioses, templos y Faraones, vol. 1, Barcelona, Folio.

Baines, John. 2001. "Egyptian Letters of the New Kingdom as Evidence for Religious Practice", en Journal of Ancient Near Eastern Religions, vol. 1, n¹, Leiden, pp. 1-31.

Banton, Michael. 1965. Roles: An Introduction to the Study of Social Relations, London, Tavistock. 
Bard, Kathryn. 1997. "Urbanism and the Rise Of Complex Society And The Early State In Egypt", en Manzanilla, Linda (ed.), Emergence and Change in Early Urban Societies, New York, Plenum, pp. 5986.

Bard, Kathryn. 2017. "Political Economies of Predynastic Egypt and the Formation of the Early State", en Journal of Archaeological Research, vol. 25, no 1, New York, pp. 1-36.

Basílico, Susana \& Lupo, Silvia (eds.). 2006. Tell el-Ghaba, norte de Sinaí, Egipto. Alimentación, producción e intercambio, Buenos Aires, Dunken.

Bennett, James Edward. 2019. The Archaeology of Egypt in the Third Intermediate Period, Cambridge, Cambridge University Press.

Bickel, Susanne. 2003. "'Ich spreche ständig zu Aton...': zur Mensch-Gott-Beziehung in der Amarna Religion", en Journal of Ancient Near Eastern Religions, vol. 3, Leiden, pp. 23-45.

Binford, Lewis. 1981. "Behavioral Archaeology and the 'Pompeii Premise'", en Journal of Anthropological Research, vol. 37, Albuquerque, NM, pp. 195-208.

Boschung, Dietrich \& Jan N. Bremmer (eds.). 2015. The Materiality of Magic (Morphomata 20), Paderborn, Wilhelm Fink.

Braudel Fernand. 1958. "Histoire et Sciences sociales: La longue durée”, en Annales. Economies, sociétés, civilisations, année $13^{\mathrm{e}}$, no 4, Paris, pp. 725-753.

Braudel, Fernand. 1987 (1949). El Mediterráneo y el mundo mediterráneo en la época de Felipe II, México, Fondo de Cultura Económica.

Bussmann, Richard. 2011. "Seals and Seal impressions from Hierakonpolis", en Egyptian Archaeology. The Bulletin of the Egypt Exploration Society, vol. 38, London, pp. 17-19.

Cabrera, Rodrigo \& Salem, Leila. 2020. “Archivos, palimpsestos y materialidad funeraria. Una discusión en Asiriología y Egiptología a partir de los Estudios de la Cultura Material”, Cuadernos de la Facultad de Humanidades y Ciencias Sociales. Universidad Nacional de Jujuy, no 57, San Salvador de Jujuy, pp. 147-175.

Calomino, Eva A. 2019. "Los small finds de Tell el-Ghaba (Norte de Sinaí, Egipto). Una propuesta teóricometodológica para analizar los hallazgos especiales en contextos domésticos entre los siglos $\mathrm{X}$ y VII a.C.", en Actas de la I Jornada en Investigación y Docencia sobre el Cercano Oriente Antiguo, 2 y 3 de noviembre de 2017, Facultad de Humanidades y Ciencias de la Educación, Universidad Nacional de La Plata, La Plata, pp. 1-10. Disponible en: http://www.memoria.fahce.unlp.edu.ar/trab_eventos/ev.13178/ev.13178.pdf. (Consultado el 10 de diciembre de 2020)

Calomino, Eva A. 2020. "Note per lo studio di 'risultati speciali' in contesti domestici. Small finds di Tell el-Ghaba (Nord Sinai, Egitto)", en VRBS. Studi sulla romanità antica e tardoantica, vol. 1, Roma, pp. 65-73. Disponible en: https://bd4232eb-d6ad-4cc4-be2214a486dec195.filesusr.com/ugd/73cfe9_56a4d947ac5f4975a95b6e4efac7dfea.pdf. (Consultado el 10 de diciembre de 2020)

Calomino, Eva A., Scaro, Agustina \& Lupo, Silvia. 2017. "Contextos, actividades y funciones en una estructura privada de Tell el-Ghaba (Norte de Sinaí, Egipto): el Edificio B", en Burgos Bernal, Laura, 
Pérez Largacha, Antonio \& Vivas Sainz, Inmaculada (coords.), Congreso Ibérico de Egiptología (Colección Estudios 157), Cuenca, Universidad de Castilla/La Mancha, p. 157.

Cione, Alberto. 2006. "Fishes from Tell el-Ghaba", en Fuscaldo, Perla (ed.), Tell el-Ghaba II. A Saite settlement in North Sinai, Egypt (Argentine Archaeological Mission 1995-2004), Vol. II: Studies (Colección Estudios 5), Buenos Aires, Instituto Multidisciplinario de Historia y Ciencias HumanasConsejo Nacional de Investigaciones Científicas y Técnicas, pp. 102-136.

Colla, Elliott. 2007. Conflicted Antiquities. Egyptology, Egyptomania, Egyptian Modernity, Durham/London, Duke University Press.

Crawford, Osbert. 1953. Archaeology and the Field, London, Phoenix House Ltd.

Davies, Norman de Garis. 1933. The Tomb of Neferhotep at Thebes, New York, Metropolitan Museum of Art.

Deetz, James. 1977. In Small Things Forgotten: The Archaeology of Early American Life, New York, Anchor Press.

Ebert, James. 1992. Distributional Archaeology, Albuquerque, University of New Mexico Press.

Frankfort, Henri, Fairman, Herbert Walter \& Pendlebury, John Devitt Stringfellow. 1933. The City of Akhenaten, Part 2, The North Suburb and the Desert Altars: the Excavations at Tell el Amarna during the Season 1926-1932, London, Egypt Exploration Society.

Fuscaldo, Perla (ed.). 2005. Tell el-Ghaba I. A Saite Settlement in North Sinai, Egypt (Argentine Archaeological Mission 1995- 2004). Vol I. The Catalogue (Colección Estudios 5), Buenos Aires, CONICET.

Fuscaldo, Perla (ed.). 2006. Tell el-Ghaba II. A Saite Settlement in North Sinai, Egypt (Argentine Archaeological Mission 1995- 2004). Vol II. Studies (Colección Estudios 5), Buenos Aires, CONICET.

García Sánchez, Jorge. 2014. Breve historia de la arqueología, Madrid, Nowtilus.

Gardiner, Alan \& Arthur E. P. Brome Weigall. 1913. A Topographical Catalogue of the Private Tombs of Thebes, London, Bernard Quaritch.

Giddy, Lisa. 1999. The Survey of Memphis III. Kom Rabi'a: the New Kingdom and Post-New Kingdom Objects, London, The Egypt Exploration Society/Cromwell Press.

Hartwig, Melinda. 2004. Tomb Painting and Identity in Ancient Thebes: 1419-1372 BCE, Turnhout, Brepols.

Gillespie, Susan D. 2007. "When is a House?", en Beck, Robin (ed.), The Durable House: House Society Models in Archaeology (Occasional Paper 35), Carbondale, Center for Archaeological Investigations, pp. 25-50.

lamarino, María Laura. 2018. "Evidencia material y culto a deidades tradicionales en Amarna", en Brancaglion, Antonio \& Chapot, Gisela (eds.), Semana de Egiptologia do Museu Nacional (SEMNA) (Estudos de Egiptologia V), Rio de Janeiro, Seshat-Laboratório de Egiptologia do Museu Nacional Editora e Klínē, pp. 106-121.

Kemp, Barry, Anna Stevens \& Paul Buckland. 2010. Busy Lives at Amarna: Excavations in the Main City (Grid 12 and the House of Ranefer, N49. 18), London, Egypt Exploration Society. 
Lacovara, Peter. 1997. The New Kingdom Royal City, London/New York, Kegan Paul International.

Lucas, Gavin. 2010. "Time and the Archaeological Archive", en Rethinking History. The Journal of Theory and Practice, vol. 14, no 3, London, pp. 343-359.

Lupo, Silvia (ed.). 2015. Tell el-Ghaba III. A Third Intermediate-early Saite Period in the Egyptian Eastern Delta. Excavations 1995-1999 and 2010 in areas I, II, VI and VIII (BAR International Series 2756), Oxford, Archaeopress.

Lupo, Silvia, Crivelli Montero, Eduardo, Kohen, Claudia \& Calomino, Eva A. 2017. "Building B, a Domestic Construction at Tell el-Ghaba, North Sinai", en Rosati, Gloria \& Guidotti, M. Cristina (eds.), Proceedings of the XI International Congress of Egyptologists. Florence Egyptian MuseumFlorence, 2330 August 2015 (Archaeopress Egyptology 19), Oxford, Archaeopress, pp. 351-356.

Lupo, Silvia, Calomino, Eva A. \& Scaro, Agustina. 2019. “El Edificio B de Tell el-Ghaba como un caso de estudio para repensar la dinámica de la unidad doméstica en el Antiguo Egipto", en Trabajos de Egiptología, vol. 10, Tenerife, pp. 201-216.

Manniche, Lise. 2001. "Funerary Cones", en Redford, Donald (ed.), The Oxford Encyclopedia of Ancient Egypt I, Oxford, Oxford University Press, pp. 565-567.

Manzi, Liliana \& Sánchez, Julián. 2007. "Bloques de distintas procedencias alojados en la tumba de Neferhotep (TT49), el-Khokha (Tebas occidental, Egipto)", en Actas del I Congresso Internacional da Sociedade de Arqueología Brasileira / XIV Congresso da SAB/III Encontro do IPHAN e Arqueólogos, Florianópolis, Sociedade de Arqueología Brasileira, pp. 95-107.

Manzi, Liliana. 2009-2010. "Intervenciones y registro de daños en la tumba de Neferhotep, la colina de el-Khokha, Tebas occidental, Egipto”, en Avances. Revista del Área Artes, no. 16, Córdoba, pp. 187201.

Manzi, Liliana. 2012. "La jerarquización del espacio a través de la distribución de tumbas privadas en Tebas Occidental, Egipto", en Manuel de Araújo, Luís \& das Candeias Sales, José (eds.), Novos Trabalhos de Egiptologia Ibérica, vol. 1, Lisboa, Instituto Oriental e Centro de História da Faculdade de Letras da Universidade de Lisboa, pp. 637-655.

Manzi, Liliana. 2016. “Monumentalización e evocación en el paisaje de Tebas occidental, Egipto”, en Revista Mundo Antigo. Dossiê Egiptologia, vol. 5, no 9, Rio de Janeiro, pp. 191-205.

Manzi, Liliana. 2018. "Personas y lugares en el paisaje de Tebas Occidental, Egipto", en Brancaglion, Antonio \& Chapot, Gisela (eds.), Semana de Egiptologia do Museu Nacional (SEMNA) (Estudos de Egiptologia V), Rio de Janeiro, Seshat-Laboratório de Egiptologia do Museu Nacional Editora e Klínē, pp. 90-105.

Meskell, Lynn. 2004. Object Worlds in Ancient Egypt: Material Biographies Past and Present, Oxford, Berg Publishers.

Miller, Daniel. 2005. “Materiality. An Introduction”, en Miller, Daniel (ed.), Materiality, Durham/London, Duke University Press, pp. 1-50.

Mitchell, Timothy. 1988. Colonising Egypt, Berkerley/Los Angeles/London, University of California Press. Molina, Manuel (ed.) 2002-. Base de Datos de Textos Neosumerios. Disponible en: http://bdts.filol.csic.es 
Molina, Manuel. 2008. "The Corpus of Neo-Sumerian Tablets: an Overview", en Garfinkle, Steven J. \& Johnson, John Cale (eds.), The Growth of an Early State in Mesopotamia: Studies in Ur III Administration. Proceedings of the First and Second Ur III Workshops at the $49^{\text {th }}$ and $51^{\text {st }}$ Rencontre Assyriologique Internationale, London July 10, 2003 and Chicago July 19, 2005, (Biblioteca del Próximo Oriente Antiguo 5), Madrid, Consejo Superior de Investigaciones Científicas, pp. 19-53.

Molina, Manuel. 2016. "Archives and Bookkeeping in Southern Mesopotamia during the Ur III Period", en Comptabilités, vol. 8, Lille. Disponible en: http://journals.openedition.org/comptabilites/1980. (Consultado el 28 de diciembre de 2020)

Molina, Manuel. 2020. "The Looting of Ur III Tablets after the Gulf Wars", en Sommerfeld, Walter (ed.), Dealing with Antiquity: Past, Present \& Future. Proceedings of the $63^{\text {rd }}$ Rencontre Assyriologique Internationale held at Marburg (Alter Orient und Altes Testament. Veröffentlichungen zur Kultur und Geschichte des Alten Orients und des Alten Testaments 460), Münster, Ugarit-Verlag, pp. 323-352.

Moreno García, Juan Carlos. 2014. "The Cursed Discipline? The Peculiarities of Egyptology at the Turn of the Twenty-First century", en Carruthers, William (ed.), Histories of Egyptology: Interdisciplinary Measures, London/New York, Routledge, pp. 50-63.

Moreno García, Juan Carlos. 2015. "Un mito tenaz: el Egipto antiguo o el paraíso perdido en la obra de los egiptólogos de finales del siglo XIX y comienzos del siglo XX", en Da Riva, Rocío \& Vidal, Jordi (eds.), Descubriendo el Antiguo Oriente. Pioneros y arqueólogos de Mesopotamia y Egipto a fines del s. XIX y principios del s. XX, Barcelona, Bellaterra, pp. 103-122.

Nelson, Richard C. 1976. Pisan-dub-ba Texts from the Sumerian Ur III Dynasty, University of Minnesota, Tesis para optar por al grado de Doctor.

Nielsen, Axel. 1987. "Trampling the Archaeological Record: an Experimental Study", en American Antiquity, vol. 53, no 3, Washington, pp. 483-503.

Nielsen, Axel. 1991. "Se hace camino al andar. Estudio experimental sobre patrones de fractura en material cerámico sometido a pisoteo", en Shincal, vol. 3, no 2, San Fernando del Valle de Catamarca, pp. 116-139.

Peet, Thomas Eric \& Woolley, C. Leonard. 1923. The City of Akhenaten, I: Excavations of 1921 and 1922 at el-'Amarna, London, Egypt Exploration Society.

Pendlebury, John Devitt Stringfellow. 1951. The City of Akhenaten: Part III: The Central City and the Official Quarters. The Excavations at Tell-El-Amarna during the Seasons 1926-1927 and 1931-1936, London, Egypt Exploration Society.

Pereyra, María Violeta, Manzi, Liliana \& Broitman, Livia. 2013. “La Tumba Tebana 49 y su propietario, en el paisaje sacralizado del occidente tebano, Egipto”, en Arqueología, vol. 19, Buenos Aires, pp. 103123.

Pereyra, María Violeta, Cerezo, María Eugenia \& Fresquet, Inés. 2007. "El aporte de los registros fragmentarios para la reconstrucción de la historia de una tumba egipcia", en Actas del I Congresso Internacional da Sociedade de Arqueología Brasileira/XIV Congresso da SAB/III Encontro do IPHAN e Arqueólogos, Florianópolis, Sociedade de Arqueología Brasileira, pp. 1-15.

Pereyra, María Violeta, Di Antonio, Maria Giorgia, Carniel, Maria Violeta \& Menozzi, Oliva. 2015. "II complesso funerario di Neferhotep (Luxor): una lunga storia di 'riutilizzi'. Progetto, metodologie, tecnologie, scavo e protocolli archeometrici integrati", en Frankfurter elektronische Rundschau zur 
Altertumskunde, vol. 28, Flensburg, pp. 17-62. Disponible en: http://www.ferajournal.eu/index.php/ojs-fera/article/view/156. (Consultado el 20 de diciembre de 2020)

Pinch, Geraldine. 1994. Magic in Ancient Egypt, London, British Museum Press.

Politis, Gustavo. 2000. "La formación de sitios de sitios de cazadores-recolectores en las Tierras Bajas Sudamericanas: un caso de estudio etnoarqueológico", en Durán Coirolo, Alicia \& Bracco Boksa, Roberto (eds.), Arqueología de las Tierras Bajas, Montevideo, Ministerio de Educación y Cultura, Comisión Nacional de Arqueología, pp. 250-266.

Porter, Bertha \& Moss, Rosalind. 1970. Topographical Bibliography of Ancient Egyptian Hieroglyphic Texts, Reliefs, and Paintings. I. The Theban Necropolis, Part 1. Private Tombs, Oxford, Griffith Institute/Ashmolean Museum.

Randall, Henry John. 1934. “History in the Open Air", en Antiquity, vol. 8, № 29, Durham, pp. 5-23.

Rzepka, Sławomir, Wodzińska, Anna, Malleson, Claire, Hudec, Jozef, Jarmużek, Łukasz, Misiewicz, Krzysztof, Małkowski, Wiesław \& Bogacki, Miron. 2011. "New Kingdom and the Third Intermediate Period in Tell el-Retaba: Results of the Polish-Slovak Archaeological Mission, Seasons 2009-2010", en Ägypten und Levante/Egypt and the Levant, vol. 21, Wien, pp. 129-184.

Sahlins, Marshall. 1972. Stone Age Economics, New York, de Gruyter.

Said, Edward. 2002 (1978). Orientalismo, Barcelona, Debolsillo.

Saleh, Mohamed. 1977. Three Old Kingdom Tombs at Thebes, Mainz am Rhein, Philipp von Zabern.

Schiffer, Michael. 1987. Formation Processes of the Archaeological Record, Albuquerque, The University of New Mexico Press.

Sharer, Robert \& Ashmore, Wendy. 2002. Archaeology: Discovering Our Past, Boston, Mc Graw-Hill.

Shaw, Ian. 1984. "Ring Bezels at el-Amarna", en Kemp, Barry (ed.), Amarna reports I, London, Egypt Exploration Society, pp. 124-132.

Simpson, Caroline. 2003. "Modern Qurna-Pieces of an Historical Jigsaw”, en Strudwick, Nigel \& Taylor, John (eds.), Present and Past in the Theban Necropolis, London, The British Museum Press, pp. 244249.

Spence, Kate. 2015. "Ancient Egyptian Houses and Households: Architecture, Artefacts, Conceptualization and Interpretation", en Müller, Miriam (ed.), Household Studies in Complex Societies. (Micro)Archaeological and Textual Approaches, Chicago, University of Chicago Press, pp. 83-99.

Stein, Julie K. 1987. "Deposits for Archaeologists", en Schiffer, Michael (ed.), Advances in Method and Theory, vol. 11, New York, Academic Press, pp. 337-395.

Steinkeller, Piotr. 2003. "Archival Practices in Babylonia in the Third Millenium", en Brosius, Maria (ed.), Ancient Archives and Archival Traditions: Concepts of Record-Keeping in the Ancient World, Oxford, Oxford University Press, pp. 37-58.

Steinkeller, Piotr. 2004. "The Function of Written Documentation in the Administrative Praxis of Early Babylonia", en Hudson, Michael \& Wunsch, Cornelia (eds.), Creating Economic Order, RecordKeeping, Standardization, and the Development of Accounting in the Ancient Near East, International Scholars Conference on Ancient Near Eastern Economies 4, Bethesda, CDL, pp. 64-88. 
Stern, Nicola. 1994. "The Implications of Time-Averaging for Reconstructing the Land-Use Patterns of Early Tool-Using Hominids", en Journal of Human Evolution, vol. 27, no 1-3, Amsterdam, pp. 89-105.

Stevens, Anna. 2006. Private Religion at Amarna. The Material Evidence, Oxford, Archeopress.

Stevens, Anna. 2015. Achaeology of Amarna, Oxford, Oxford Handbooks Online.

Strudwick, Nigel \& Strudwick, Helen. 1999. Thebes in Egypt: A Guide to the Tombs and Temples of Ancient Luxor, London, British Museum Press.

Strudwick, Nigel \& Taylor, John (eds.). 2003. Present and Past in the Theban Necropolis, London, The British Museum Press.

Sullivan, Alan. 2008. "Time Perspectivism and the Interpretive Potential of Palimpsests: Theoretical and Methodological Considerations of Assemblage Formation History and Contemporaneity", en Holdaway, Simon \& Wandsnider, LuAnn (eds.), Time in Archaeology: Time Perspectivism Revisited, Salt Lake City, UT, University of Utah Press, pp. 31-45.

Tilley, Christopher. 2008. "Phenomenological Approaches to Landscape Archaeology", en David, Bruno \& Thomas, Julian (eds.), Handbook of Landscape Archaeology, California, Left Coast Press, pp. 271276.

Trigger, Bruce. 1992 (1989). Historia del pensamiento arqueológico, Barcelona, Editorial Crítica.

Tsouparopoulou, Christina. 2013. "Reflections on Paratextual Markers and Graphic Devices in Ur III Administrative Documents", en Textual Cultures, vol. 8, no 2, Buffalo, pp. 1-14.

van der Spek, Kees. 2011. The Modern Neighbors of Tutankhamun: History, Life, and Work in the Villages of the Theban West Bank, Cairo/New York, The American University in Cairo Press.

Vivant Denon, Dominique. 2005 (1802). Sin Mañana. Viaje al Bajo y Alto Egipto durante las campañas del general Bonaparte, Madrid, Atalanta.

Wengrow, David. 2006. The Archaeology of Early Egypt. Social Transformations in North-East Africa, 10,000-2650 BC, Cambridge, Cambridge University Press.

Wilson, Penelope \& Bertini, Louise. 2011. Sais I: The Ramesside-Third Intermediate Period at Kom Rebwa, London, Egypt Exploration Society.

Winter, Irene J. 1997. "Art in Empire: The Royal Image and the Visual Dimensions of Assyrian Ideology", en Parpola, Simo \& Whiting, Robert M. (eds.), Assyria 1995: Proceedings of the $10^{\text {th }}$ Anniversary Symposium of the Neo-Assyrian Text Corpus Project, Helsinki, September 7-11, 1995, Helsinki, NeoAssyrian Text Corpus Project, pp. 359-381. 